\title{
Long Standing Temporomandibular Joint Dislocation: A Case Report
}

\author{
Dr. Dipanjal Saikia ${ }^{\mathrm{I}}$ \\ ${ }^{I}$ Associate Professor, Department of Dentistry, Assam Medical College and Hospital, Dibrugarh, Assam
}

\begin{abstract}
Dislocation of the temporomandibular joint is not an uncommon condition. It may be unilateral or bilateral and can be acute, recurrent and rarely chronic. The pathogenesis of chronic dislocation is diverse. Manual reduction may be difficult to reduce after certain period because of fibrosis. Surgical reduction is than indicated. This paper reports a case of chronic TMJ dislocation in a dentulous patient in which conventional manual reduction with local anaesthesia, sedation or general anaesthesia with muscle relaxants failed Than elastic traction was tried which initially failed. But it was than successfully reduced when elastic traction was applied along with a specialy designed bite plate. Hence, with properly selected chronic cases for elastic traction; where path of displacement of the condyle is in a favourable path with the resultant force vector retraction path of traction; this non surgical procedure can be successfully tried for tmj reduction.
\end{abstract}

Key Words: Bite Plate, Elastic Traction, Non surgical TMJ reduction, TMJ dislocation.

\section{Introduction}

Dislocation of the temporomandibular joint is not an uncommon condition. It represents $3 \%$ of all reported dislocated joints in the body [1] . It Occurs when the condyle is dislocated from the joint into an abnormal position. It may be unilateral or bilateral. Various classifications of TMJ dislocation have been reported based on the direction of displacement and location of the condylar head [2,3]. Most cases of TMJ dislocation are anterior. As per duration ; dislocation of the temporomandibular joint is commonly acute, occasionally recurrent, and rarely chronic. Chronic dislocation has been arbitrarily defined as dislocation which persists for more than one month [4] .The pathogenesis of chronic dislocation is diverse. Inadequate or inability to diagnose or treat acute dislocation remains the principal underlying cause. Manual reduction may be difficult to reduce after certain period because of fibrous tissue within the glenoid fossa [5,6].Surgical reduction is than indicated. This paper reports a case of chronic dislocation of the TMJ in which conventional manual reduction with local anaesthesia, sedation or general anaesthesia with muscle relaxants failed. Than elastic traction was tried which initially failed. But it was than successfully reduced when elastic traction was applied along with a specially designed posterior bite plate.

\section{Case Report}

A 38-year-old male patient with chief complain of pain in the preauricular region and with difficulty in talking; eating and closing of the mouth came to the department of Dentistry, Assam medical college and Hospital on 28th Nov 2011. The pain occurred one night after he yawned widely and was than unable to close his mouth. Examination revealed that pain was localized in the joint areas. It was dull, throbing, and aggravated when jaw movement was done. Movement radiated the pain to nearby areas. There were no other associated symptoms such as pus discharge from oral cavity, swelling, vertigo, tinnitus or any history of similar dislocations in the past. There was no paresthesia or altered sensation in the facial area. Examination revealed bilateral dislocation of TMJ both clinically and radio graphically with Class III type occlusion. (Fig.1a,1b,1c,1d,1e).

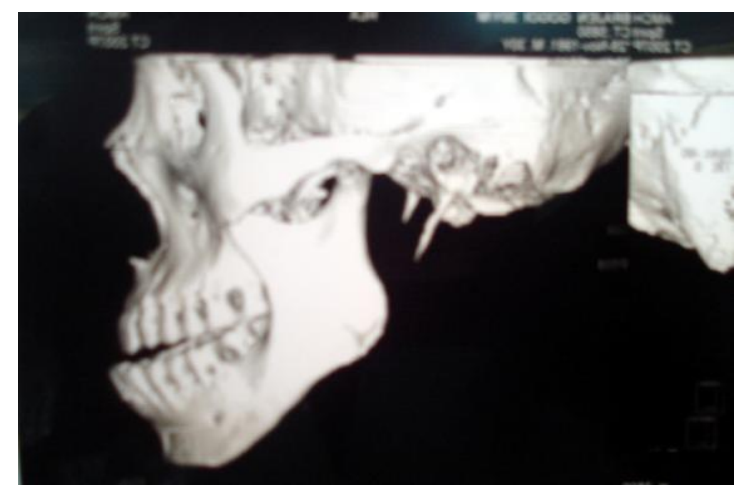

fig-1a 


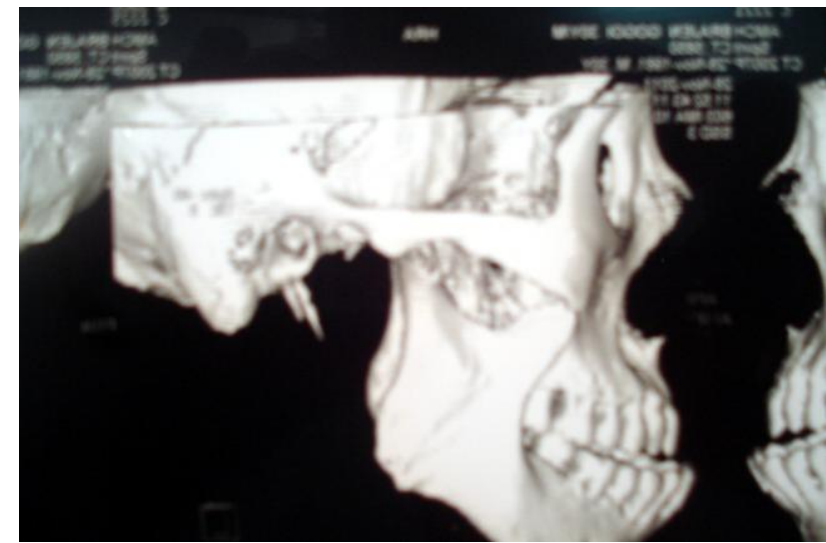

fig $1 b$

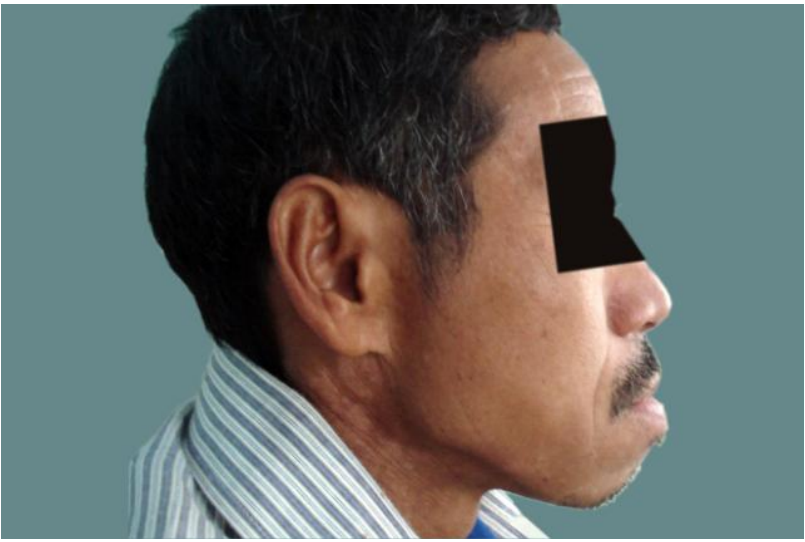

fig $1 \mathrm{c}$

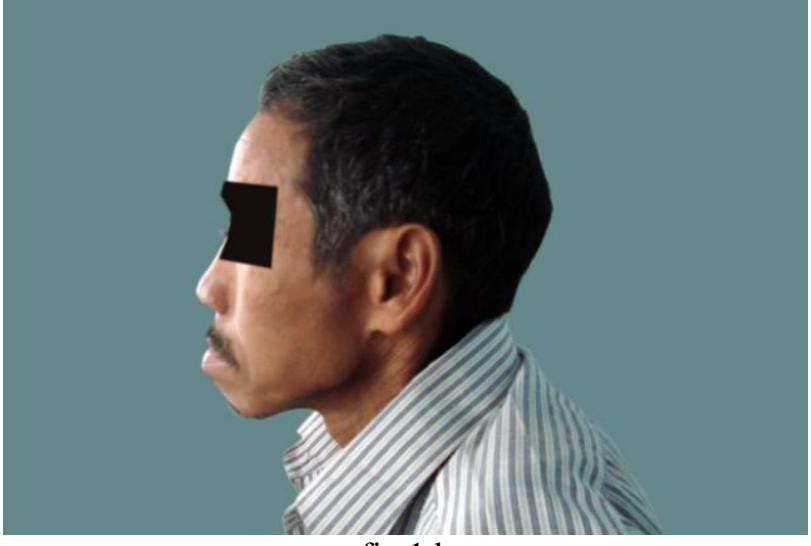

fig $1 \mathrm{~d}$

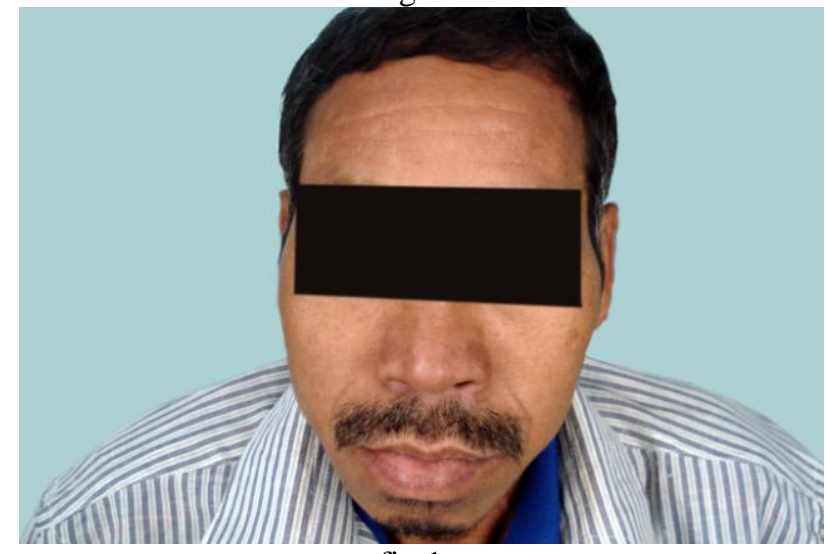

fig $1 \mathrm{e}$ 
Past medical history revealed that he had gone to a nearby hospital where he was given first aid with medications. Being not relieved for several weeks after taking treatment at his locality, he came to our department. The patient had been experiencing the pain and discomfort for the last 8 weeks. Manual reduction with local anaesthesia, sedation or general anaesthesia with muscle relaxants was unsuccessful. Patient was explained that elastic traction will be tried. If not successful; than surgical correction for dislocation will be opted. Erich arch bar were fixed to both jaws extending from molar to molar. Heavy elastic traction with class III traction on premolar area and anterior elastics were also applied. Elastics were manually made from the rubber tubing of iv drip set with $2 \mathrm{~mm}$ width. Elastic traction was changed everyday. Four days of traction did not produce any significant change. Now impressions of both the arches were taken and an upper acrylic plate with $\mathrm{C}$ clasps and posterior bite plate extending from 3rd molars to 2nd premolars on both the sides was fabricated. Bite plate was inserted and heavy elastic tractions with Class III vector on the premolar area only and anterior elastics were placed (Fig-2a ).

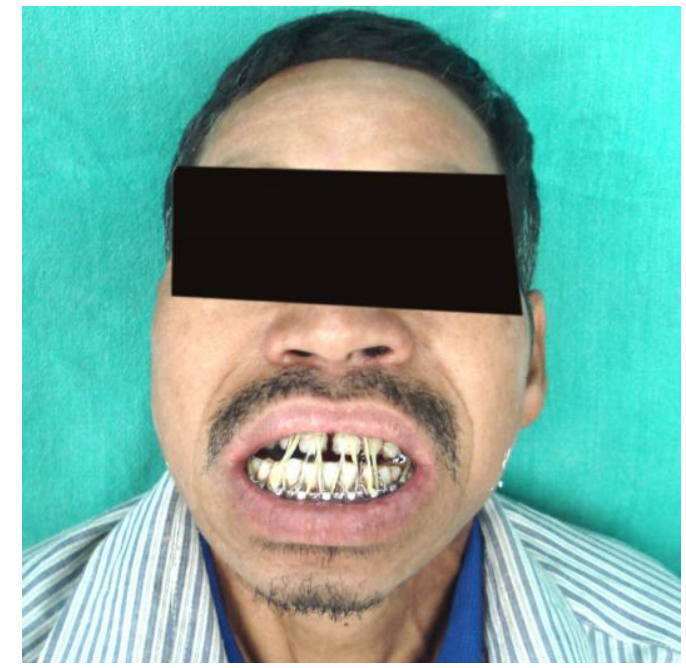

fig $2 \mathrm{a}$

Elastics were changed every day. After 4 days of elastic traction with bite plate; plate was removed and class III traction continued along with anterior elastic pull. On the seventh day from bite plate addition, occlusion had almost come back to its normal position( Fig 3a,3b).

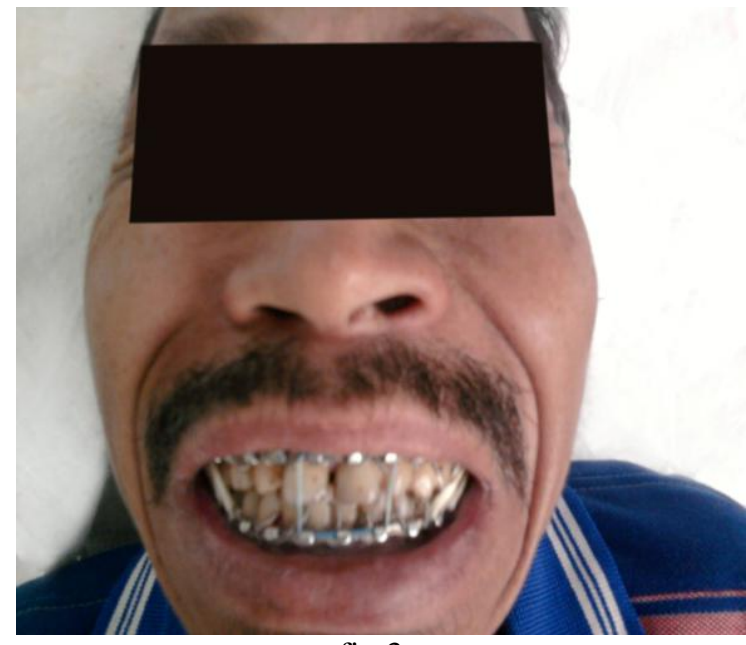

fig $3 \mathrm{a}$ 


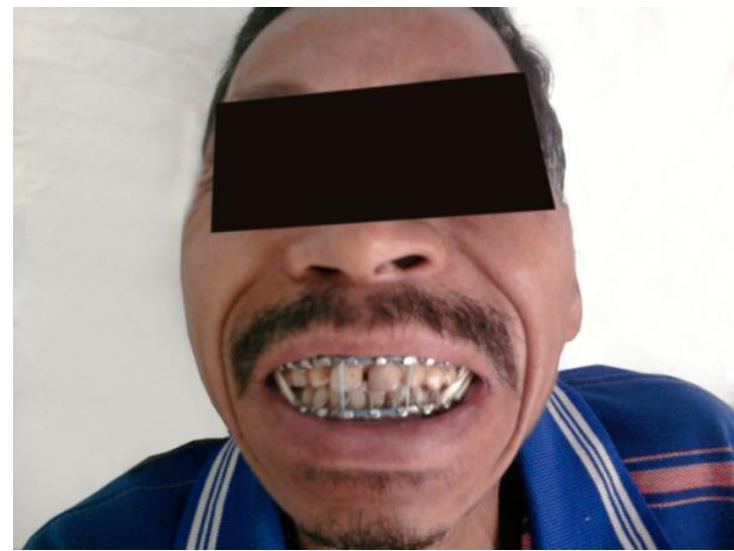

fig $3 b$

Pain was significantly less now. IMF was maintained with elastic traction for another seven days. Than elastics were removed . Initial mouth opening was restricted ( fig-4a,4b,4c).

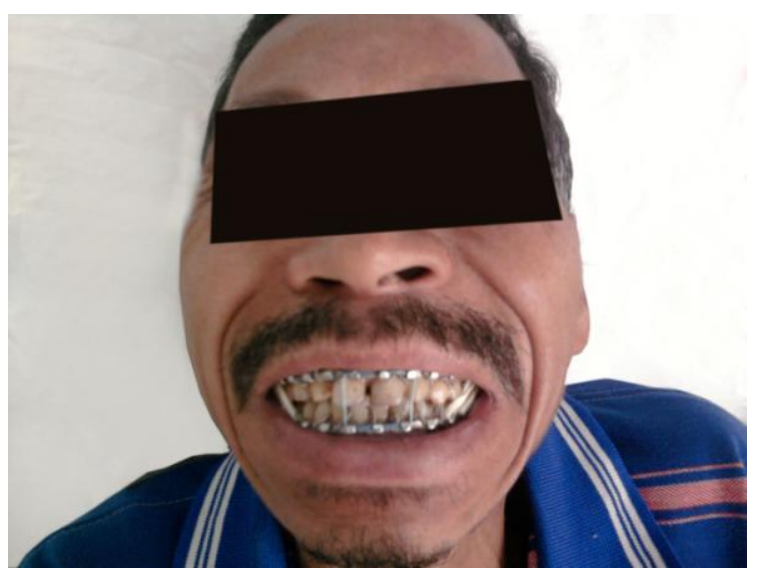

fig $4 \mathrm{a}$

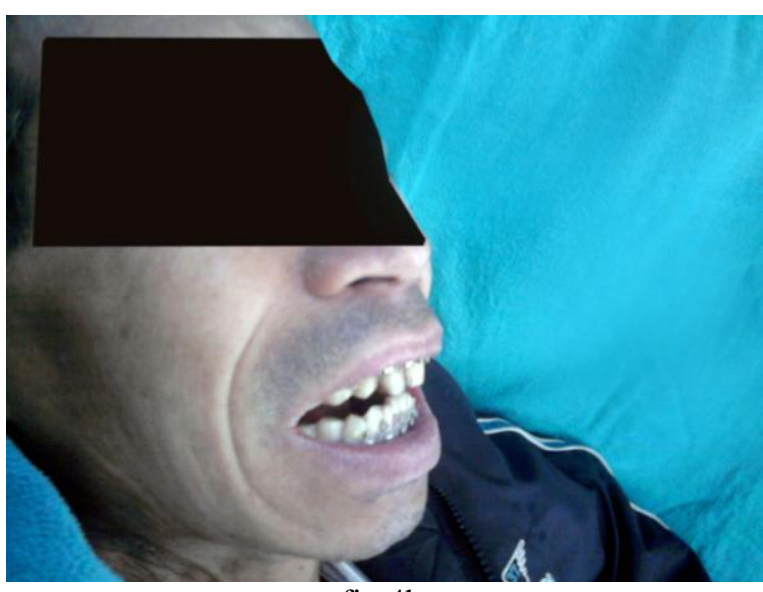

fig $4 \mathrm{~b}$ 


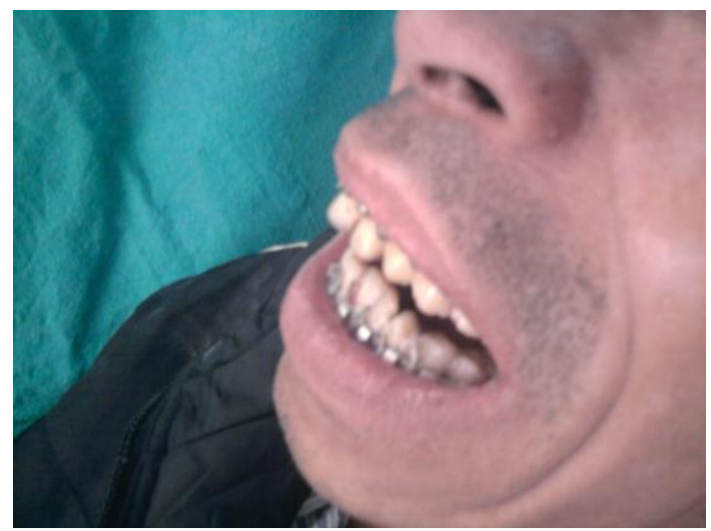

fig $4 \mathrm{c}$

Patient was discharged with the advice to continue gradual mouth opening and movements and to wear light elastics at bed time only to maintain occlusion for another one more week. He was also advised to review after 2 weeks. He was adviced to abstain from wide yawning, vigourous chewing, heavy strenous activity, heavy jerks and not to sleep laterally. After 2 weeks mouth opening improved and movements were satisfactory and pain during movements of the jaw was less. ( fig $5 \mathrm{a}, 5 \mathrm{~b}$ ) .

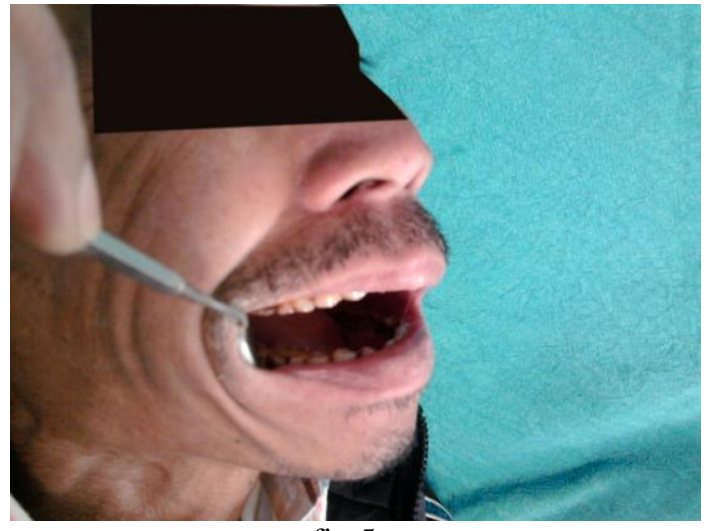

fig 5 a

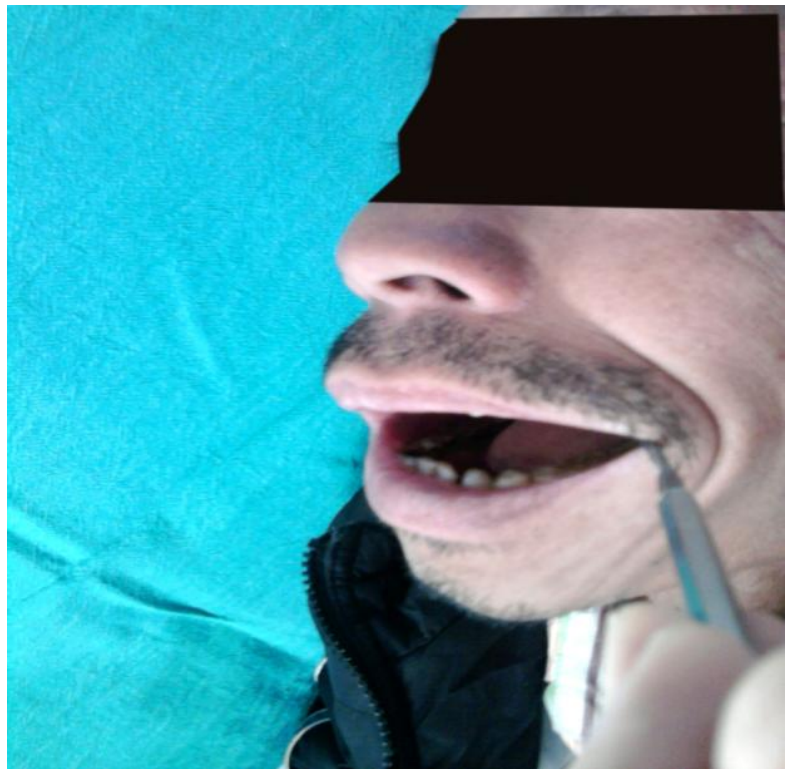

fig $5 b$

There was no relapse of dislocation. Patient again came for review after 6 months and there was no dislocation or pain in joints till than. 


\section{Discussion:}

Late presentation for dislocation treatment may be due to ignorance, improper medical service provided, financial restrictions, distance from a specialized healthcare facility. Joint overextension may be caused by yawning, vomiting, screaming, laughing, or during an epileptic seizure.[7] Asymptomatic TMJ disorders in apparently young healthy patient may act as a predisposing factor and partly explain how yawning could trigger off joint dislocation [8].

Dislocation of the condyle causes muscle spasm and if prolonged leads to soft tissue fibrosis and ingrowth of soft tissue into the glenoid fossa making reduction difficult.

Typically TMJ dislocation is managed either conservatively or surgically and is influenced by whether the condition presented was acute, recurrent or chronic. Manual reduction with or without anaesthesia/ analgesia is preferred for acute dislocation $[9,10,11]$.

Chronic bilateral dislocation of the temporomandibular joint (TMJ) is most likely to occur in the elderly, edentulous, and medically compromised patient [12] . Chronic (prolonged) cases are more difficult to manage manually [8]. Most agree that the longer the dislocation, the more difficult the reduction of the condyle [13]. When dislocation has persisted for 4-12 weeks ; surgical treatment is opted [13]. Various surgical tecniques have been proposed which are best determined by proper individual clinical evaluation and with minimum postoperative morbidity.

The case presented here shows that nonsurgical reduction still has a place for some chronic long standing dislocation cases. In absence of teeth, elastic traction can be given with dentures [12]. In this case ; initial elastic traction with the posterior teeth acting as pivot for the lever force generated in the mandible to pull the condyle down and back into the fossa was not sufficient. With incorporation of bite plate; condyle could be pulled down more which aided in sliding of the condyle back down the eminence slope ; into the fossa (Fig 6 ) .

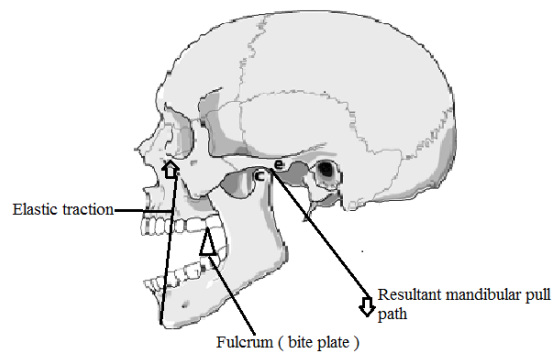

fig 6

Hence, with proper selection of chronic cases ; where displacement of the condyle is in a favourable path with the resultant force vector retraction path of traction ; elastic traction can be successfully tried for non surgical TMJ reduction

\section{References:}

[1]. R. M. Aleman Navas, M. G. Martınez Mendoza: 'Inverse' temporomandibular joint dislocation. Int. J. Oral Maxillofac. Surg. 2011; 40: $877-879$

[2]. Allen FJ, Young AH. Lateral displacement of the intact mandibular condyle. A report of five cases. Br J Oral Surg 1969: 7: 24-30.

[3]. Zecha JJ. Mandibular condyle dislocation into the middle cranial fossa. Int J Oral Surg 1977: 6: 141-146.

[4]. FORDYCE GL. Long-standing bilateral dislocation of the jaw. Br J Oral Surg 1965: 2:222 5.

[5] HAMMERSLEY N. Chronic bilateral dislocation of the temporomandibular joint. Br J Oral Maxillofac Surg 1986: 24: 365- 75.

[6]. LITTLER BO. The role of local anaesthesia in the reduction of long-standing dislocation of the temporomandibular joint. Br J Oral Maxillofac Surg 1980: 18: 81-5.

[7]. S Chhabra, N Chhabra. CASE REPORT: Recurrent bilateral TMJ dislocation in a 20-month-old child: A rare case presentation. Journal of Indian Society of Pedodontics and Preventive Dentistry, 2011, Volume 29, Issue 6 [p. 104-106]

[8]. V. I. Ugboko, F. O. Oginni, S. O. Ajike, H. O. Olasoji, E. T. Adebayo:A survey of temporomandibular joint dislocation: aetiology, demographics, risk factors and management in 96 Nigerian cases. Int. J. Oral Maxillofac. Surg. 2005; 34: 499-502.

[9]. Adekeye EO, Shamia RI, Cove P. Inverted L-shaped ramus osteotomy for prolonged bilateral dislocation of the temporomandibular joint. Oral Surg Oral Med Oral Pathol 1976: 41: 568-577.

[10]. Caminiti MF, Weinberg S. Chronic mandibular dislocation: the role of nonsurgical and surgical treatment. J Can Dent Assoc 1998: 64: 484-491.

[11]. Gray RJ, Davies SJ. Emergence treatment of acute temporomandibular disoders. Part 11. Dent Update 1997: 24: 186- 189.

[12]. K. Kurita, Y. Mukaida, N. Ogi, M. Toyama: Closed reduction of chronic bilateral temporomandibular joint dislocation. A case report. Int. J. Oral Maxillofac. Surg. 1996; 25: 4-423.

[13]. I-Y. Huang, C. -M. Chen, Y. -H. Kao, C. -M. Chen, C. -W. Wu: Management of longstanding mandibular dislocation. Int. J. Oral Maxillofac. Surg. 2011; 40: 810-814. 